\title{
Single top quark production and CKM matrix elements measurements with CMS
}

\author{
Agostino De lorio* on behalf of the CMS Collaboration \\ Università degli Studi di Napoli "Federico II" e INFN sezione di Napoli \\ E-mail: adeiorio@fisica.unina.it, deiorio@na.infn.it
}

\begin{abstract}
The study of single top quark inclusive production provides important insight into the electroweak processes of the standard model of elementary particles and into the structure of the proton. It also enables a direct measurement of the magnitude of the Cabibbo-Kobayashi-Maskawa (CKM) matrix elements. Among the production channels, the $t$-channel process is the dominant mechanism in proton-proton collisions at the CERN LHC accounting for approximately $70 \%$ of the total single top quark production cross section at center-of-mass energy of $13 \mathrm{TeV}$. The state of the art of on single top quark $t$-channel measurements performed by the CMS experiment, and their impact on our knowledge of the CKM matrix elements and top quark couplings will be presented.
\end{abstract}

European Physical Society Conference on High Energy Physics - EPS-HEP2019 -

10-17 July, 2019

Ghent, Belgium

${ }^{*}$ Speaker. 


\section{Single top processes}

In proton-proton collisions at a center-of-mass energy of $13 \mathrm{TeV}$ at the Large Hadron Collider (LHC), the single-top-quark production could happen in three different ways:

- $t$-channel: This is the process with the largest cross section. A light-flavour quark q from one of the colliding protons interacts with a b-quark by exchanging a space-like virtual $\mathrm{W}$ boson, producing a top quark (t-quark) and a recoiling light-flavour quark $\mathrm{q}^{\prime}$, called the spectator quark.

- $\mathrm{tW}$ associate production: This is the process with the second largest cross section. A gluon interacts with an initial b-quark by exchanging a virtual b-quark, producing a $\mathrm{t}$-quark and a W boson.

- $s$-channel: This is the process with the smallest cross section. A quark-antiquark pair annihilates to produce a time-like virtual $\mathrm{W}$ boson, which decays to a t-quark and a b-antiquark.

The single top production processes are particularly indicated to perform the measurement of the Cabibbo-Kobayashi-Maskawa element $\left|\mathrm{V}_{\mathrm{tb}}\right|$.

This document describes the measurement of the most dominant process, $t$-channel, that has cross section of:

$$
\sigma_{t-\mathrm{ch} . t+\bar{t}}^{\text {theo }}=217.0_{-4.6}^{+6.6}(\mathrm{scale}) \pm 6.2\left(\mathrm{PDF}+\alpha_{S}\right) \mathrm{pb}
$$

\section{CMS experiment}

The central feature of the CMS apparatus is a superconducting solenoid of $6 \mathrm{~m}$ internal diameter, providing a magnetic field of 3.8 T. Within the solenoid volume are a silicon pixel and strip tracker, a lead tungstate crystal electromagnetic calorimeter (ECAL), and a brass and scintillator hadron calorimeter (HCAL), each composed of a barrel and two endcap sections. Forward calorimeters extend the pseudorapidity $(\eta)$ coverage provided by the barrel and endcap detectors. Muons are measured in the range $|\eta|<2.4$ using gas-ionisation detectors embedded in the steel flux-return yoke outside the solenoid. A more detailed description of the CMS detector, together with a definition of the coordinate system used and the relevant kinematic variables, can be found in Ref. [1].

\section{Analysis strategy}

\subsection{Physics objects selection}

The final-state topology in the $t$-channel is characterized by the presence of exactly one isolated charged lepton and a b jet from the top quark decay, as well as a light-flavoured jet produced in the forward region. Potential gluon splitting in the initial state leads to a second b quark (Fig. 1) that recoils against the top quark and thus has generally a softer $p_{\mathrm{T}}$ spectrum and a broader $|\eta|$ 


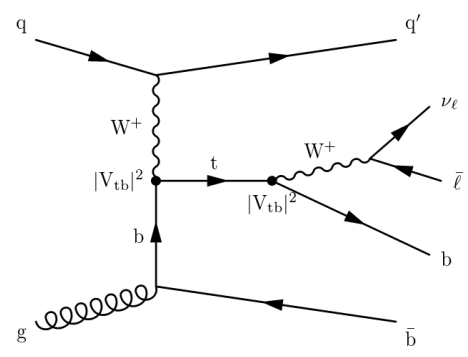

Figure 1: Feynman diagram for the single-top $t$-channel production.

\begin{tabular}{lcll}
\hline Object & $\#$ & Requirement & \\
\hline Tight muon & 1 & $p_{\mathrm{T}}>26 \mathrm{GeV} / \mathrm{c}$ & $|\eta|<2.4 \quad I_{\text {rel }}^{\mu}<0.06$ \\
Loose muon & 0 & $p_{\mathrm{T}}>10 \mathrm{GeV} / \mathrm{c}$ & $|\eta|<2.4 \quad I_{\text {rel }}^{\mu}<0.2$ \\
Tight electron & 1 & $E_{\mathrm{T}}>35 \mathrm{GeV}$ & $|\eta|<2.1 \quad I_{\text {rel }}^{\mathrm{e}}<0.0588$ (B) $\quad I_{\text {rel }}^{\mathrm{e}}<0.0571$ (E) \\
Loose electron & 0 & $E_{\mathrm{T}}>15 \mathrm{GeV}$ & $|\eta|<2.5 \quad I_{\text {rel }}^{\mathrm{e}}<0.2$ \\
Jet & $2(3)$ & $E_{\mathrm{T}}>40 \mathrm{GeV}$ & $|\eta|<4.7$ \\
b-Jet & $1(2)$ & $E_{\mathrm{T}}>40 \mathrm{GeV}$ & $|\eta|<2.4 \quad$ CSVv2 $\geq 0.9535$ \\
\hline
\end{tabular}

Table 1: Details of the objects required by the analysis [2].

distribution compared to the $\mathrm{b}$ quark produced in the top-quark decay. The acceptance of events with two $b$ jets reconstructed in the final state is therefore expected to be small.

The detailed description of the objects used in the analysis [2], the number of the object used and the requirements they have to satisfy are reported in Table 1.

\subsection{Multiple regions definitions}

Following the selection reported the signal region is found to have:

- 1 light jet in high $\eta$ region

- Lepton from the $\mathrm{W}$ boson

- Missing momentum in the transverse plane $(v)$

- $\mathrm{b}$ jest from the top decay

- $\mathrm{b}$ from the gluon splitting out of selection

Several backgrounds are included when the selection is performed. They are the top pair production ( $(\mathrm{t})$, the vector boson + jets $(\mathrm{V}+\mathrm{HF})$, the single-top $s$-channel, the tW associate production and the QCD multijets. Three orthogonal regions defined accordingly to the number of jets and $\mathrm{b}$-jet present in each event. The region with 2 jets, one of them passing the b-tagging requirement is the signal region. Regions with three jets are used as control regions to constraint the uncertainty on the background normalization. 


\begin{tabular}{ll}
\hline Variable & Description \\
\hline Light jet $|\eta|$ & Absolute value of the pseudorapidity of the light-quark jet \\
Dijet mass & Invariant mass of the two selected jets \\
Top quark mass & Invariant mass of the top quark reconstructed from lepton, neutrino and the b-tagged jet \\
$\Delta R$ (lepton, b jet) & $\Delta R$ between the momentum vectors of the lepton and the b-tagged jet \\
$\cos \theta^{*}$ & Cosine of the angle between the lepton and the light-quark jet in the rest frame of the top quark \\
Jet $p_{\mathrm{T}}$ sum & Scalar sum of the transverse momentum of the two jets \\
$m_{\mathrm{T}}^{\mathrm{W}}$ & Transverse mass of the W boson \\
$p_{\mathrm{T}}^{m i s s}$ & Missing momentum in the transverse plane of the event \\
$\Delta R$ (light jet, b jet) & $\Delta R$ between the momentum vectors of the light-quark jet and the b-tagged jet \\
Lepton $|\eta|$ & Absolute value of the pseudorapidity of the selected lepton \\
$\mathrm{W}$ boson $|\eta|$ & Absolute value of the pseudorapidity of the reconstructed W boson \\
Light jet mass & Invariant mass of the light-quark jet \\
\hline
\end{tabular}

Table 2: Variables used in the multivariate analysis [2].

\subsection{Estimation QCD contribution}

The production of multijets through QCD processes has a huge cross section. On the other hand, only a small fraction of these events mimics the lepton+jets final state of the applied event selection and the selection efficiency for QCD multijet events is therefore tiny. This implies that to obtain a sample with enough statistic one should produce really huge MC samples. Another way is to extract the shape of the distribution directly from the data by reverting the isolation requirement on the lepton. The right normalization is obtained by fitting the $m_{\mathrm{T}}^{\mathrm{W}}\left(p_{\mathrm{T}}^{\text {miss }}\right)$ variable for the muon (electron) in the isolated region. Finally the QCD sample is depleted by applying the requirement $m_{\mathrm{T}}^{\mathrm{W}}>50 \mathrm{GeV}$ for muon channel and $p_{\mathrm{T}}^{\text {miss }}>30 \mathrm{GeV}$ for the electron channel.

\subsection{Multivariate analysis and fit procedure}

Several kinematic variables are reconstructed from the objects selected in order to discriminate between the signal and the different backgrounds. These variables are reported in detail in Table 2.

The kinematic variables are used to train a Boost Decision Trees discriminator for both muon and electron channel. The distribution of the discriminators used in the analysis are reported in Fig. 2 for both the muon and electron channels.

Finally a simultaneous Maximum Likelihood fit is performed to the 12 categories ( 2 lepton flavour $\times 3$ region $\times 2$ electric charges) defined and the value for the total cross section is:

$$
\sigma_{t-\text { ch. }, t+\bar{t}}^{\text {theo }}=219 \pm 2(\text { stat }) \pm 6(\text { prof }) \pm 35(\text { sig }- \text { mod }) \pm 6(\text { lumi }) \text { pb }
$$

\section{Results}

The absolute value of $\mathrm{V}_{\mathrm{tb}}$ is extracted from the measurement of the cross section by using the following formula:

$$
\left|f_{\mathrm{LV}} \mathrm{V}_{\mathrm{tb}}\right|=\sqrt{\frac{\sigma_{t-\mathrm{ch}, t+\bar{t}}}{\sigma_{t-\mathrm{ch} . t+\bar{t}}^{\text {theo }}}}
$$



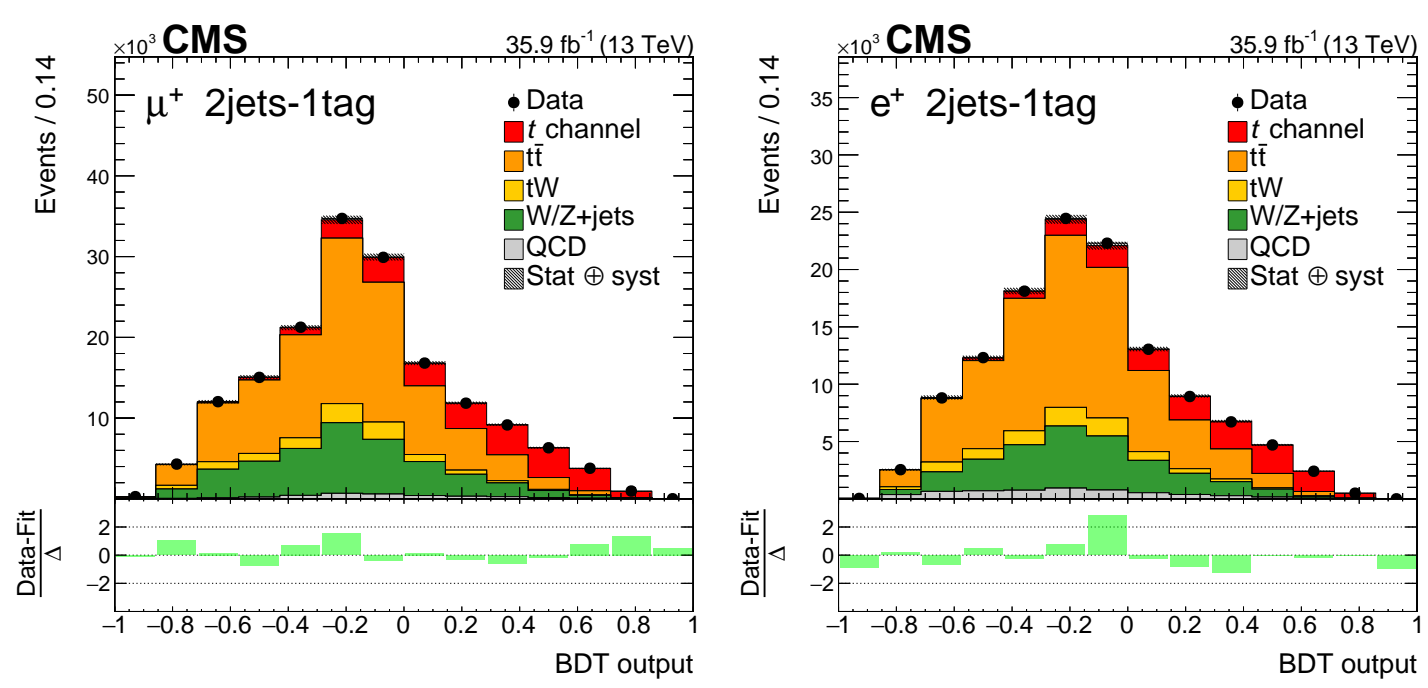

Figure 2: Distribution of the discriminators in the signal region for both the muon and electron channels [2].

where $f_{\mathrm{LV}}$ is the anomalous form factor that takes the possible presence of an anomalous $\mathrm{Wtb}$ coupling into account. Within the $\mathrm{SM}, f_{\mathrm{LV}}=1$.

The result obtained by this analysis is:

$$
\left|f_{\mathrm{LV}} \mathrm{V}_{\mathrm{tb}}\right|=1.00 \pm 0.08 \text { (meas) } \pm 0.02 \text { (theo) }
$$

This result is in agreement with value obtained from the combination of all the single-top-quark production cross section measurements with the full Run-I data [3]:

$$
\left|f_{\mathrm{LV}} \mathrm{V}_{\mathrm{tb}}\right|=1.02 \pm 0.04(\text { meas }) \pm 0.02(\text { theo })
$$

\section{References}

[1] S. Chatrchyan et al. [CMS Collaboration], "The CMS Experiment at the CERN LHC," JINST 3, S08004 (2008). doi:10.1088/1748-0221/3/08/S08004

[2] A. M. Sirunyan et al. [CMS Collaboration], "Measurement of the single top quark and antiquark production cross sections in the $t$-channel and their ratio in proton-proton collisions at $\sqrt{s}=13 \mathrm{TeV}$," arXiv:1812.10514 [hep-ex].

[3] M. Aaboud et al. [ATLAS and CMS Collaborations], "Combinations of single-top-quark production cross-section measurements and $\left|f_{\mathrm{LV}} V_{t b}\right|$ determinations at $\sqrt{s}=7$ and $8 \mathrm{TeV}$ with the ATLAS and CMS experiments," JHEP 1905, 088 (2019) doi:10.1007/JHEP05(2019)088 [arXiv:1902.07158 [hep-ex]]. 